\title{
Dielectronic satellite lines and double layers in solar flares
}

\author{
E. Dzifč́áková1 ${ }^{1}$ M. Karlický1 ${ }^{1}$, and J. Dudík ${ }^{1,2}$
}

\author{
1 Astronomical Institute of the Academy of Sciences of the Czech Republic, Fričova 298, 25165 Ondřejov, Czech Republic \\ e-mail: elena@asu.cas.cz \\ 2 Department of Astronomy, Physics of the Earth and Meteorology, Faculty of Mathematics, Physics and Informatics, \\ Comenius University, Mlynská Dolina F2, 84248 Bratislava, Slovak Republic
}

Received 4 October 2012 / Accepted 3 December 2012

\begin{abstract}
Context. Particle acceleration during solar flares results in departures of the distribution of particle energies from the Maxwellian distribution. Apart from the high-energy tail, the bulk of the distribution was recently also found to be significantly affected, due, e.g., to the presence of double layers.

Aims. We investigate the influence of several proposed non-Maxwellian distribution functions on the X-ray flare line spectra. The distribution functions considered are sharply peaked and include the $n$-distribution, the moving Maxwellian distribution, and the distribution formed in strong double layers in the flaring plasma.

Methods. Synthetic Si XIId-Si XIV spectra involving allowed and dielectronic transitions at 5-6 $\AA$ are calculated numerically. The parameters chosen for the calculations correspond to the impulsive phase of solar flares, as inferred by previous authors.

Results. The Si XIId $\lambda 5.56 / \mathrm{Si}$ XIII $\lambda 5.68$ and Si XIId $\lambda 5.82 / \mathrm{Si}$ XIII $\lambda 5.68$ ratios depend on the relative number of electrons at energies corresponding to the formation of the Si XIId lines. Therefore, these ratios increase with the increasing narrowness of the peak of the electron distribution function. The highest ratios are achieved for the distribution formed in double layers, while the moving Maxwellian distribution is less likely to reproduce the observed enhancement of Si XIId intensities. However, the ratio of the allowed Si XIV $\lambda 5.22 / \mathrm{Si}$ XIII $\lambda 5.68$ transitions depends on the ionization equilibrium. This ratio is very small for the double-layer distribution. Combination of the double-layer distribution with a Maxwellian distribution with the same mean energy significantly enhances this ratio, while keeping the Si XIId intensities sufficiently increased to explain the characteristics of the observed spectra.

Conclusions. These results support the presence of double layers in the plasma during impulsive phase of solar flares.
\end{abstract}

Key words. Sun: X-rays, gamma rays - Sun: flares - atomic processes - radiation mechanisms: non-thermal

\section{Introduction}

Solar flares are dynamic manifestations of magnetic energy release accompanied by particle acceleration (e.g., Fletcher et al. 2011; Holman et al. 2011; Lin 2011; Zharkova et al. 2011; Raymond et al. 2012). The most common consequence of electron acceleration is the presence of high-energy power-law tail (e.g., Lin \& Hudson 1971; Holman et al. 2003; Veronig et al. 2010), i.e., an enhanced number of electrons with energies up to several tens of $\mathrm{MeV}$. However, departures from the equilibrium Maxwellian distribution in solar flares were also observed at much lower energies, several $\mathrm{keV}$, corresponding to the excitation energies of X-ray lines, e.g., of He-like Fe (Seely et al. 1987) or Si (Dzifčáková et al. 2008).

That the departures from Maxwellian distribution affect line intensities, especially of X-ray lines, has already been proposed by, e.g., Gabriel \& Phillips (1979). These authors showed that the high-energy tail would lead to a decrease in the observed Fe XXIVd/Fe XXV ratio because of the enhanced excitation of the Fe XXV resonance line due to the high-energy tail. However, an opposite situation was reported by Seely et al. (1987). These authors proposed that a distribution with a slope steeper than the Maxwellian distribution at energies corresponding to the formation of Fe XXIVd dielectronic satellite lines could explain the observed enhanced excitation of these lines. To do that, they introduced an analytical, parametric $n$-distribution

$f(\mathcal{E}, n) \mathrm{d} \mathcal{E}=\mathcal{B}_{n} \frac{2}{\sqrt{\pi}\left(k_{\mathrm{B}} T\right)^{3 / 2}}\left(\frac{\mathcal{E}}{k_{\mathrm{B}} T}\right)^{\frac{n-1}{2}} \mathcal{E}^{1 / 2} \mathrm{e}^{-\mathcal{E} / k_{\mathrm{B}} T} \mathrm{~d} \mathcal{E}$, where $\mathcal{B}_{n}=\sqrt{\pi} /(2 \Gamma(n / 2+1))$ is the normalization constant, $k_{\mathrm{B}}$ is the Boltzmann constant, and $n \in\langle 1, \infty)$ and $T$ are the parameters of the distribution. For $n=1$, the distribution is Maxwellian. The mean energy $\langle\mathcal{E}\rangle=3 / 2 k_{\mathrm{B}} \tau$, where $\tau=$ $(n / 2+1) T$, is the pseudo-temperature.

Following this result, Dzifčáková et al. (2008) and Kulinová et al. (2011) showed that the Si XIId/Si XIII ratios observed during impulsive phases of several solar flares observed by the RESIK instrument (Sylwester et al. 2005, also Fig. 1) can be explained neither by an iso-thermal nor by a multi-thermal plasma with a Maxwellian distribution. They diagnosed values of $n=11$ or higher. Furthermore, Kulinová et al. (2011) showed that highest values of $n$ are observed during the impulsive phase and coincide with the presence of type III radio bursts. This means that the $n$-distributions should be connected with the beam and return-current system in the flare plasma (Dzif̌́áková \& Karlický 2008; Karlický et al. 2012). Including the $n$-distribution also significantly improves the goodness-of-fit of the RHESSI data at energies of $4 \mathrm{keV}$ or more (Kulinová et al. 2011). During the decay phase, the RESIK spectra can be well fitted by the Maxwellian distribution of isothermal plasma (Fig. 1 bottom), indicating equilibration and cooling of the plasma after cessation of the particle acceleration processes.

Karlický et al. (2012) investigated the physical meaning of the $n$-distributions in connection to the beam - returncurrent system in solar flares. They found that the Maxwellian distribution with a velocity drift $v_{0}$ ("moving Maxwellian") closely mimics the high-energy slope of the $n$-distribution 

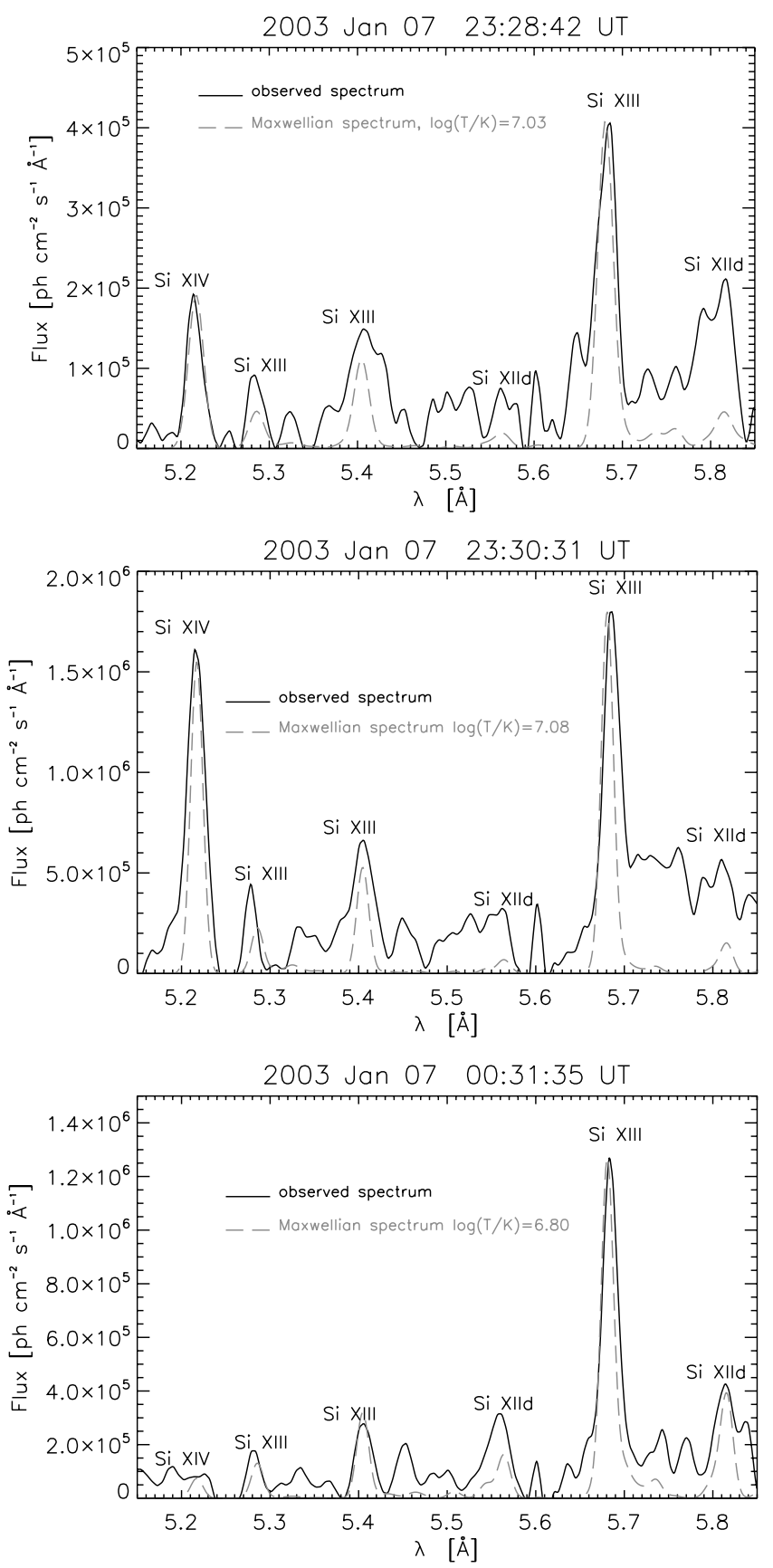

Fig. 1. X-ray spectra observed by the RESIK instrument during impulsive (top and middle) and decay (bottom) phase of an M4.9 flare on January 07, 2003 (Dzif̌ćáková et al. 2008). Synthetic Maxwellian isothermal Si spectra having the same Si XIV $\lambda 5.22 / \mathrm{Si}$ XIII $\lambda 5.68$ ratio are indicated by the grey dashed lines. Note that the RESIK observations contain a number of unidentified lines.

when expressed as a function of electron energy,

$f\left(\mathcal{E}, v_{0}\right) \mathrm{d} \mathcal{E}=\frac{\sinh \left(2 \frac{v_{0}}{v_{\mathrm{T}}} \sqrt{\frac{\mathcal{E}}{k_{\mathrm{B}} T}}\right)}{\sqrt{\pi} k_{\mathrm{B}} T v_{0} / v_{\mathrm{T}}} \mathrm{e}^{-\mathcal{E} / k_{\mathrm{B}} T-\left(v_{0} / v_{\mathrm{T}}\right)^{2}} \mathrm{~d} \mathcal{E}$,

where $v_{\mathrm{T}}$ is the thermal velocity of the plasma. To reach values of $n=11$ or higher, $v_{0} / v_{\mathrm{T}}$ has to be greater than 1 . This means that the moving Maxwellian distribution is unstable, mainly due to the Buneman instability. That the observed departures from the Maxwellian distribution last several minutes (Kulinová et al. 2011, Figs. 4, 7, and 9 therein) means that the true distribution
Table 1. Strongest Si lines observed in the fourth channel of RESIK.

\begin{tabular}{lcc}
\hline \hline Ion & $\lambda[\AA]$ & Transition \\
\hline Si XIV & 5.22 & $1 \mathrm{~s}^{2} \mathrm{~S}_{1 / 2}-3 \mathrm{p}^{2} \mathrm{P}_{1 / 2,3 / 2}$ \\
Si XIII & 5.28 & $1 \mathrm{~s}^{2}{ }^{1} \mathrm{~S}_{0}-1 \mathrm{~s} 5 \mathrm{p}^{1,3} \mathrm{P}_{1}$ \\
Si XIII & 5.40 & $1 \mathrm{~s}^{2}{ }^{1} \mathrm{~S}_{0}-1 \mathrm{~s} 4 \mathrm{p}^{1,3} \mathrm{P}_{1}$ \\
Si XIII & 5.68 & $1 \mathrm{~s}^{1} \mathrm{~S}_{0}-1 \mathrm{~s} 3 \mathrm{p}^{1,3} \mathrm{P}_{1}$ \\
Si XIId & 5.56 & $1 \mathrm{~s}^{2} 2 \mathrm{p}^{2} \mathrm{P}_{1 / 2,3 / 2}-1 \mathrm{~s} 2 \mathrm{~s} 4 \mathrm{p}^{2} \mathrm{D}_{3 / 2,5 / 2}$ \\
Si XIId & 5.82 & $1 \mathrm{~s}^{2} 2 \mathrm{p}^{2} \mathrm{P}_{1 / 2,3 / 2}-1 \mathrm{~s} 2 \mathrm{~s}\left({ }^{3} \mathrm{P}\right) 3 \mathrm{p}^{2} \mathrm{D}_{3 / 2,5 / 2}$ \\
\hline
\end{tabular}

in the flaring plasma should be stable. By using the 3D particlein-cell simulations of a flare loop containing electron beam, reflected beam, and background plasma with return current, Karlický (2012) showed that the formation of double layers (DL) in flare plasma can lead to a stable peak of the distribution function, closely mimicking the $n$-distribution at the required energy range. This peak is superimposed on the broken power-law distribution. The DL is a spatially very narrow structure of the strong electric field parallel to the magnetic field lines. Based on the numerical simulations (Karlický 2012), it was found that only electrons entering into this layer can be accelerated. Thus, the Maxwellian distribution (on the low-potential side of DL) after an acceleration in this layer results in the distribution peak (on the high-potential side of DL), which can be approximated by a moving half-Maxwellian distribution, i.e., a distribution that is Maxwellian in $v_{x}$ and $v_{y}$, and also $v_{z}>0$, but zero for $v_{z}<0$, where $z$ is the coordinate along the flare loop. The moving halfMaxwellian distribution is hereafter referred to as the distribution in DL or simply the "DL-distribution".

While having the same gradient $\mathrm{d} f / \mathrm{d} \mathcal{E}$ at the energies of few $\mathrm{keV}$, where the dielectronic satellite lines are formed, the $n$-distribution, moving Maxwellian, and DL-distributions differ by orders of magnitude in the low-energy end (Fig. 2 top). These differences can affect the recombination rates and subsequently the diagnostics of non-Maxwellian plasma during the impulsive phase of solar flares. In this paper, we investigate the influence of these distributions on the formation of flare X-ray spectra containing the dielectronic satellite lines of $\mathrm{Si}$.

\section{Method}

We calculate the Si synthetic spectra at 5-6 to the fourth channel of the RESIK instrument. The relevant Si transitions are listed in Table 1. To calculate the intensities of these lines for the considered distributions, we use the method described by Dzifčáková et al. (2008). The ionization, recombination, and excitation rates are given by the integral product of the distribution function and the corresponding cross sections (e.g., Dzifčáková 2000; Dzifčáková \& Tóthová 2007). We used the atomic data available in the CHIANTI database (Dere et al. 1997, 2009; Landi et al. 2012). The ionization rates are obtained from the work of Dere (2007), while the radiative and dielectronic recombination rates for the ions studied here are obtained from the works of Colgan et al. (2004), Badnell (2006a,b), and Bautista \& Badnell (2007). The excitation rates are calculated using the method of Dzifčáková \& Mason (2008) with the atomic data of Wiese et al. (1966), Lin et al. (1977), Armour et al. (1979), Vainshtein \& Safronova (1978, 1980), Martin \& Zalubas (1983), Sampson et al. (1983), Drake (1986), Zhang \& Sampson (1987), Zhang et al. (1990), Aggarwal \& Kingston (1992), Chen et al. (1993), Martin et al. (1993, 1995), and Phillips et al. (2005), implemented in the CHIANTI database. 

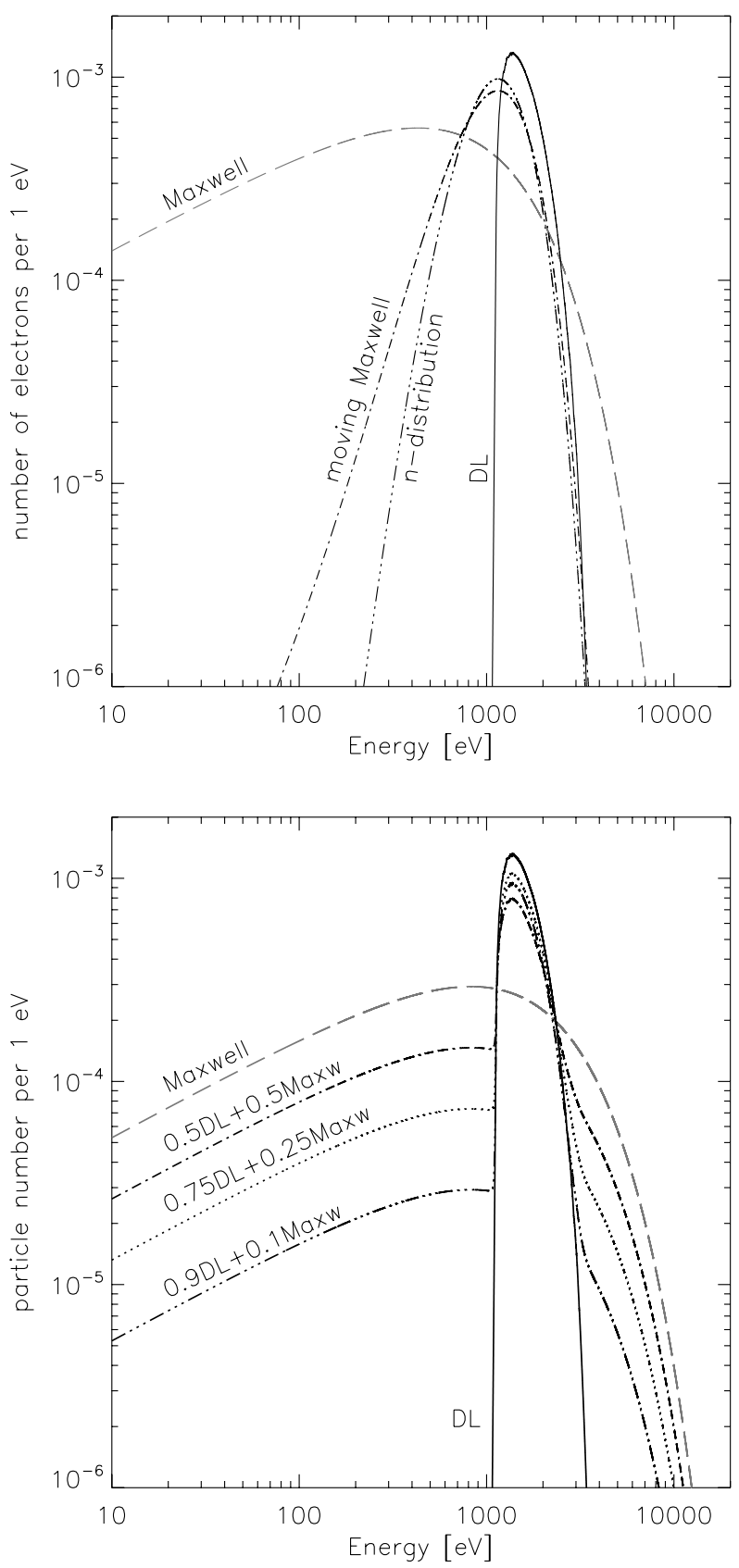

Fig. 2. Distribution functions. Top: Maxwellian, $n$-distribution, moving Maxwellian and DL-distributions. Bottom: combination of the DL-distribution $(\log (\tau)=7.28)$ with Maxwellian distribution $(\log (T)=$ 7.28). The relative weights are indicated.

We do not use the newest available excitation rates or $A$ values for Si XIII and Si XIV (Aggarwal \& Keenan 2010), since these contain calculations only for $\log (T / K) \leq 7$, which is less than the $\log (\tau / K)$ inferred from RESIK observations. Furthermore, the calculations of Aggarwal \& Keenan (2010) differ by only several percent from the ones implemented in the CHIANTI database.

To calculate the synthetic spectra, we consider the Maxwellian distribution, the $n$-distribution (Eq. (1)), moving Maxwellian distribution (Eq. (2)) and the DL-distribution. The DL-distribution (moving half-Maxwellian distribution) is computed numerically. Based of the physical considerations for formation of the DL-distributions and the results of numerical simulations of DL, the Maxwellian distribution of $10^{6}$ numerical electrons in the $3 \mathrm{D}$ velocity space is produced first, using the random-number generator. Then to all numerical electrons with the velocity component $v_{z}>0$, the drift velocity $v_{0}$ is added. Finally, this moving half-Maxwellian 3D velocity distribution is recalculated into the energy distribution, giving thus the DL-distribution.

The considered distributions, normalized to unity, are shown in Fig. 2 top. With the exception of the Maxwellian distribution, they have the same $\mathrm{d} f / \mathrm{d} \mathcal{E}$. The DL-distribution is the narrowest and thus has the highest peak. The $n$-distribution with the same $\mathrm{d} f / \mathrm{d} \mathcal{E}$, corresponding to $n=16.3$, exhibits lower mean energy, lower peak, and more low-energy electrons. The moving Maxwellian distribution has the peak even lower, with further increased number of low-energy electrons.

In addition to these distributions, we consider a weighted combination of Maxwellian and DL-distributions. I.e., we want to mimic the results of the particle-in-cell simulations of Karlický (2012). For simplicity, we assume that the DL-distribution and the background Maxwellian distribution have the same mean energies, corresponding to the $\log \left(T_{\text {Maxw }}\right)=$ $\log \left(\tau_{\mathrm{DL}}\right)=7.28$. This value is the highest one derived from RESIK observations by Dzifčáková et al. (2008) and Kulinová et al. (2011).

We note again that the observed RESIK spectra are already well-fitted by the $n$-distribution (Dzifčáková et al. 2008). Therefore, in this paper we only focus on identifying the physical process forming the distribution function, which in turn produces the same type of flare spectra as the observed ones.

In the calculation of the synthetic spectra, the flaring plasma is assumed to be in the ionization equilibrium. This is because the ionization timescales are shorter than the observed changes in $\tau$ and the distribution function (Kulinová et al. 2011).

\section{Results}

First, we studied the effect of the distribution on the ratio of allowed Si XIV $\lambda 5.22 / \mathrm{Si}$ XIII $\lambda 5.68$ lines together with the ratios involving dielectronic Si XIId $\lambda 5.56 / \mathrm{Si}$ XIII $\lambda 5.68$ and Si XIId $\lambda 5.82 / \mathrm{Si}$ XIII $\lambda 5.68$ lines. The results are shown in Fig. 3, using full black lines for the considered distribution. The dashed grey lines denote the corresponding Maxwellian spectrum having the same Si XIV/Si XIII ratio. The value of $\log (T)$ for this corresponding Maxwellian spectrum is indicated in each figure.

For the DL-distribution, the Si XIId/Si XIII ratios are very high (full line in Fig. 3 top). This is caused by the narrow peak of the DL-distribution, resulting in an increased number of particles at the energies of $\approx 2 \mathrm{keV}$ where the dielectronic $\mathrm{Si}$ XIId lines are excited. We note that the Si XIId/Si XIII ratios are independent of the relative ion abundance. This is not true for the $\mathrm{Si}$ XIV/Si XIII ratio. This ratio is strongly dependent on the ionization equilibrium. The calculated ratio for the DL-distribution is very low (Fig. 3 top), which is caused mainly by the ionization equilibrium, since the narrow peak of the DL-distribution results in narrow peaks of the ion abundances as function of $\langle\mathcal{E}\rangle$ or $\tau$ (cf. Dzifčáková 1998).

For these reasons, the wider peak of the $n$-distribution causes a decrease in the SiXIId/Si XIII ratios and an increase in the Si XIV/Si XIII ratio (Fig. 3 middle). The even wider peak of the moving Maxwellian distribution results in further changes in all of the considered ratios (Fig. 3 bottom). Nevertheless, the spectra for all considered distributions show increased Si XIId/Si XIII ratios with respect to the Maxwellian spectra having the same Si XIV/Si XIII ratio. However, the increase of the dielectronic satellite lines is most pronounced with the increasing 

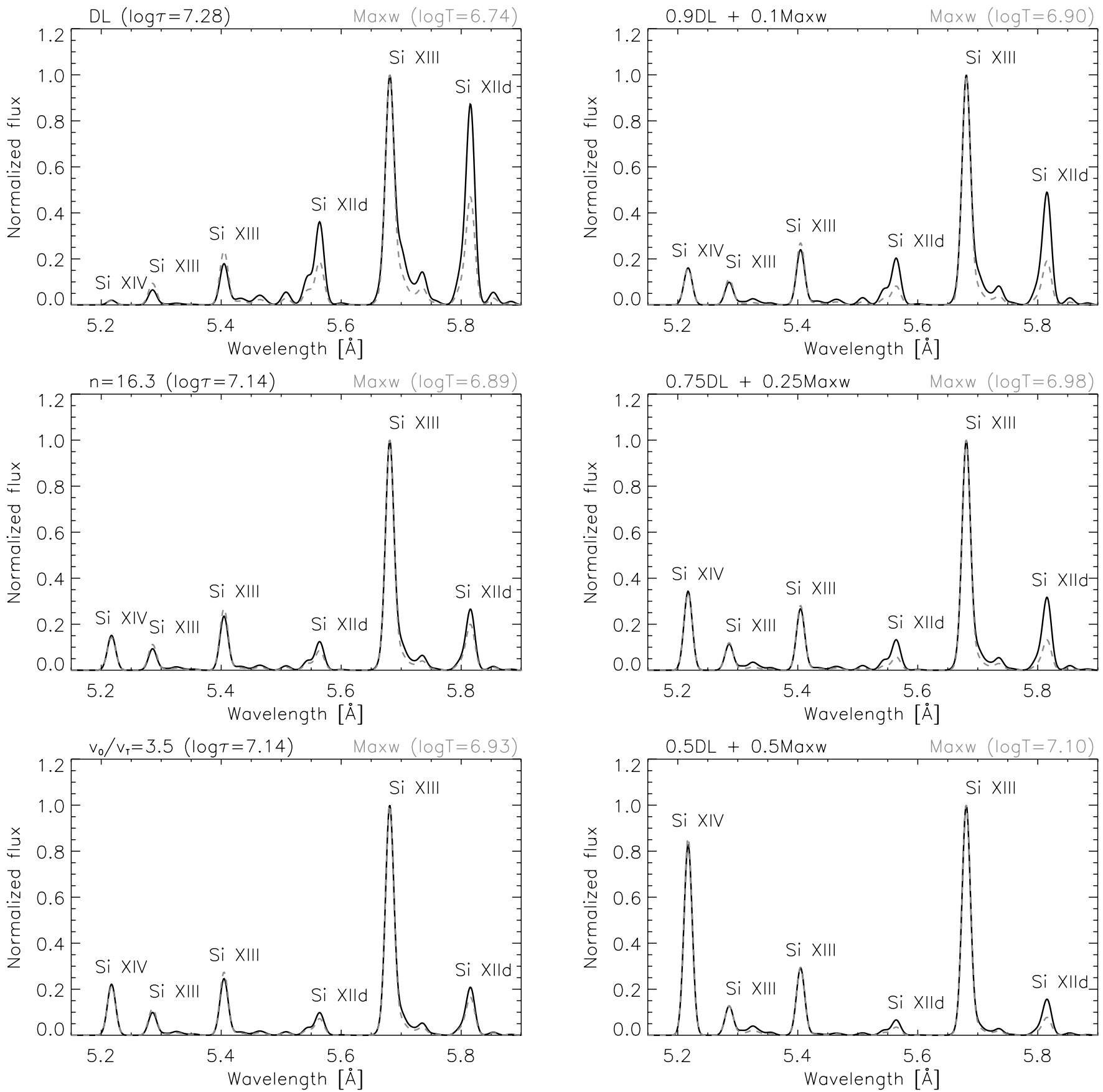

Fig. 3. Synthetic spectra (full black lines) for the DL-distribution (top), the $n$-distribution (middle) and the moving Maxwellian distribution (bottom). Dashed grey lines show Maxwellian spectra having the same Si XIV/Si XIII ratio. The parameters of each distribution are indicated in each figure.

narrowness of the peak of the distribution, with largest departures from Maxwellian spectra arising for the DL-distribution. Therefore, it is unlikely that the moving Maxwellian distribution would produce a good fit to the observations, where the Si XIId/Si XIII ratios are much more enhanced with respect to the Maxwellian spectra (Dzifčáková et al. 2008; Kulinová et al. 2011).

The combination of the DL-distribution with the Maxwellian distribution results in a strong increase of the Si XIV/Si XIII ratio with the increasing portion of the Maxwellian distribution (Fig. 4). Moreover, the increase in the Si XIId/Si XIII ratios with

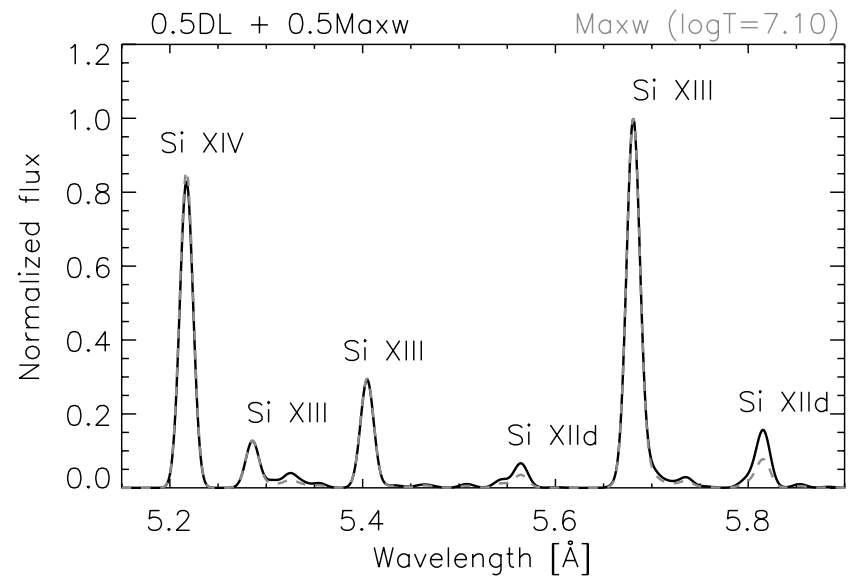

Fig. 4. Synthetic spectra (full black lines) for the combination of the DL-distribution with the Maxwellian distribution (Fig. 2 bottom), both having $\log (\tau)=7.28$. The relative weights of each distribution are indicated in each figure. Dashed grey lines show Maxwellian spectra having the same Si XIV/Si XIII ratio. The parameters of each distribution are indicated in each figure.

respect to the corresponding purely Maxwellian spectra (dashed lines in Fig. 4) are significant for all considered weights. This increase is about a factor of 2 or more. Both these effects are in good agreement with the characteristics of the spectra observed by the RESIK instrument (cf. Fig. 1), exhibiting significant increase of the Si XIId/Si XIII ratios, together with the presence of a strong Si XIV resonance line during the impulsive phase of several flares (Dzifčáková et al. 2008; Kulinová et al. 2011). From the distributions considered here, we conclude that the combination of the DL-distribution with the Maxwellian 
distribution can reproduce all features of the spectra observed by the RESIK instrument. This provides strong support for the presence of DLs in solar flares.

\section{Summary and discussion}

We investigated the influence of various distribution functions on the resulting X-ray synthetic spectra of Si emitted at 5-6 under the conditions corresponding to the impulsive phase of the solar flares. The considered distributions included the parametric $n$-distributions, the analytical moving Maxwellian distribution, and the distribution formed in plasma DL. While the ratios of dielectronic-to-allowed Si XIId/Si XIII lines do not depend on the relative ion abundances, the ratio of the allowed Si XIV/Si XIII is influenced by the ionization equilibrium. The DL-distribution has the narrowest peak of all the considered distributions, resulting in highest Si XIId/Si XIII ratios, but very small values of the $\mathrm{Si}$ XIV/Si XIII ratio. Distributions with a wider peak show a decrease in the relative intensities of the Si XIId lines, accompanied by an increase in the relative intensity of the allowed Si XIV transition.

We also analyzed the more realistic situation where the observed line emission originates in both DL and thermal background plasma having the same mean energy. The addition of a thermal source leads to an increase in the Si XIV/Si XIII ratio, while keeping the Si XIId/Si XIII ratios several times higher than the purely Maxwellian spectrum with the same Si XIV/Si XIII ratio. These results are in agreement with the characteristics of the solar flare spectra observed by the RESIK instrument, showing both a strong Si XIV line and significantly enhanced Si XIId lines. We conclude that these results provide evidence for the presence of DL in the impulsive phase of solar flares, i.e., at times when particle acceleration can be inferred directly from RHESSI and radio observations. We note that the presence of DLs in the impulsive flare phase is further supported by direct observations of the DL by the FAST satellite in the terrestrial aurora, where the physical conditions are similar (Andersson et al. 2008).

The presence of a high-energy non-Maxwellian tail has only a small influence on the Si XIId/Si XIII ratios (Dzifčáková et al. 2011). For the typical relative number of particles present in the high-energy tail, as inferred from the RHESSI observations (e.g., Warmuth et al. 2009; Veronig et al. 2010; Guo et al. 2011; Lin 2011), the effect of the tail is negligible. Therefore, the increase in Si XIId excitation is indicative of the departures of the bulk of the distribution from the Maxwellian distribution (Dzifčáková et al. 2011). However, the presence of the high-energy tail can influence the Si XIV/Si XIII ratio through the ionization equilibrium. A complete diagnostic of all effects would require simultaneous observations of the high-energy tail, together with several He-like ions with the corresponding X-ray dielectronic satellite lines, to constrain the distribution function at multiple energies. This remains a challenge for future instrumentation.

Acknowledgements. This work was supported by the Grant No. 1/0240/11 of the Scientific Grant Agency, VEGA, Slovakia, and by Grants No. 209/12/1652 and 209/12/0103 of the Grant Agency of the Czech Republic, and the Bilateral Project APVV CZ-SK-0153-11 (7AMB12SK154) involving the Slovak Research and Development Agency and the Ministry of Education of the Czech Republic. J.D. also acknowledges support from Comenius University grant No. UK/11/2012. CHIANTI is a collaborative project involving the NRL (USA),
RAL (UK), MSSL (UK), the Universities of Florence (Italy) and Cambridge (UK), and George Mason University (USA). CHIANTI is a great spectroscopic database and software, and the authors are very grateful for its existence and availability.

\section{References}

Aggarwal, K. M., \& Kingston, A. E. 1992, Phys. Scr., 46, 193 Aggarwal, K. M., \& Keenan, F. P. 2010, Phys. Scr., 82, 065302

Andersson, L., Newman, D. L., Ergun, R. E., et al. 2008, Phys. Plasmas, 15, 072901

Armour, I. A., Bashkin, S., Jelley, N. A., et al. 1979, J. Phys., 1, 211

Badnell, N. R. 2006a, A\&A, 447, 389

Badnell, N. R. 2006b, ApJS, 167, 334

Bautista, M. A., \& Badnell, N. R. 2007, A\&A, 466, 755

Chen, M. H., Cheng, K. T., \& Johnson, W. R. 1993, Phys. Rev. A, 47, 3692

Colgan, J., Pindzola, M. S., \& Badnell, N. R. 2004, A\&A, 417, 1183

Dere, K. P. 2007, A\&A, 466, 771

Dere, K. P., Landi, E., Mason, H. E., Monsignori Fossi, B. C., \& Young, P. R. 1997, A\&AS, 125, 149

Dere, K. P., Landi, E., Young, P. R., et al. 2009, A\&A, 498, 915

Drake, G. W. F. 1986, Phys. Rev. A, 34, 2871

Dzifčáková, E. 1998, Sol. Phys., 178, 317

Dzifčáková, E. 2000, Sol. Phys., 196, 113

Dzif̌ćáková, E., \& Karlický, M. 2008, Sol. Phys., 250, 329

Dzifč́áková, E., \& Mason, H. E. 2008, Sol. Phys., 247, 301

Dzifčáková, E., \& Tóthová, D. 2007, Sol. Phys., 240, 211

Dzifčáková, E., Kulinová, A., Chifor, C., et al. 2008, A\&A, 488, 311

Dzifčáková, E., Homola, M., \& Dudík, J. 2011, A\&A, 531, A111

Fletcher, L., Dennis, B. R., Hudson, H. S., et al. 2011, Space Sci. Rev., 159, 19

Gabriel, A. H., \& Phillips, K. J. H. 1979, MNRAS, 189, 319

Guo, J., Liu, S., Fletcher, L., \& Kontar, E. P. 2011, ApJ, 728, 4

Holman, G. D., Sui, L., Schwartz, R. A., \& Emslie, A. G. 2003, ApJ, 595, L97

Holman, G. D., Aschwanden, M. J., Aurass, H., et al. 2011, Space Sci. Rev., 159, 107

Karlický, M. 2012, ApJ, 750, 49

Karlický, M., Dzifčáková, E., \& Dudík, J. 2012, A\&A, 537, A36

Kulinová, A., Kašparová, J., Dzifčáková, E., et al. 2011, A\&A, 533, A81

Landi, E., Del Zanna, G., Young, P. R., Dere, K. P., \& Mason, H. E. 2012, ApJ, 744,99

Lin, R. P. 2011, Space Sci. Rev., 159, 421

Lin, R. P., \& Hudson, H. S. 1971, Sol. Phys., 17, 412

Lin, C. D., Johnson, W. R., \& Dalgarno, A. 1977, Phys. Rev. A, 15, 154

Martin, I., Karwowski, J., Diercksen, G. H. F., \& Barrientos, C. 1993, A\&AS 100,595

Martin, W. C., Dalton, G. R., Fuhr, J. R., et al. 1995, in Laboratory and Astronomical High Resolution Spectra, eds. A. J. Sauval, R. Blomme, \& N. Grevesse, ASP Conf. Ser., 81, 597

Martin, W. C., \& Zalubas, R. 1983, J. Phys. Chem. Ref. Data, 12, 323

Phillips, K. J., Sylwester, J., Sylwester, B., \& Dubau, J. 2005, AGU Spring Meeting Abstracts, B8

Raymond, J. C., Krucker, S., Lin, R. P., \& Petrosian, V. 2012, Space Sci. Rev., 173, 197

Sampson, D. H., Goett, S. J., \& Clark, R. E. H. 1983, Atomic Data and Nuclear Data Tables, 29, 467

Seely, J. F., Feldman, U., \& Doschek, G. A. 1987, ApJ, 319, 541

Sylwester, J., Gaicki, I., Kordylewski, Z., et al. 2005, Sol. Phys., 226, 45

Vainshtein, L. A., \& Safronova, U. I. 1978, Atomic Data and Nuclear Data Tables, 21, 49

Vainshtein, L. A., \& Safronova, U. I. 1980, Atomic Data and Nuclear Data Tables, 25, 311

Veronig, A. M., Rybák, J., Gömöry, P., et al. 2010, ApJ, 719, 655

Warmuth, A., Holman, G. D., Dennis, B. R., et al. 2009, ApJ, 699, 917

Wiese, W. L., Smith, M. W., \& Glennon, B. M. 1966, Atomic Transition Probabilities, Hydrogen through Neon, A critical data compilation (Washington, D.C.: US Government Printing Office)

Zhang, H., \& Sampson, D. H. 1987, ApJS, 63, 487

Zhang, H. L., Sampson, D. H., \& Fontes, C. J. 1990, Atomic Data and Nuclear Data Tables, 44, 31

Zharkova, V. V., Arzner, K., Benz, A. O., et al. 2011, Space Sci. Rev., 159, 357 